\title{
Quality of life assessment in esophagectomy patients
}

\author{
Alla Alghamedi ${ }^{1,2}$, Gordon Buduhan ${ }^{2}$, Lawrence Tan ${ }^{2}$, Sadeesh Kumar Srinathan ${ }^{2}$, Joanne Sulman ${ }^{3}$, Gail \\ Darling $^{4}$, Biniam Kidane ${ }^{2}$
}

${ }^{1}$ Section of General Surgery, ${ }^{2}$ Section of Thoracic Surgery, University of Manitoba, Winnipeg, Canada; ${ }^{3}$ Factor-Inwentash Faculty of Social Work, University of Toronto, Toronto, Canada; ${ }^{4}$ Division of Thoracic Surgery, Toronto General Hospital, University of Toronto, Toronto, Canada

Contributions: (I) Conception and design: All authors; (II) Administrative support: All authors; (III) Provision of study materials or patients: All authors; (IV) Collection and assembly of data: All authors; (V) Data analysis and interpretation: All authors; (VI) Manuscript writing: All authors; (VII) Final approval of manuscript: All authors.

Correspondence to: Biniam Kidane, MD, MSc, FRCSC. 820 Sherbrook Street, GE-611, Winnipeg, R3A 1R9 Manitoba, Canada. Email: bkidane@hsc.mb.ca.

\begin{abstract}
Esophagectomy is the mainstay of curative therapy for esophageal cancer; however, it is associated with significant morbidity and mortality, with subsequent major impact on quality of life. This paper reviews the evaluation of health-related quality of life (HRQOL) in esophageal cancer patients undergoing curative intent therapy, the relationship between postoperative HRQOL and survival as well the potential utility of pre-treatment HRQOL as a prognostic tool. HRQOL assessment is valuable in helping clinicians understand the impact on patients of esophageal cancer and the various treatments thereof. HRQOL is also valuable as an end-point in studies of esophageal cancer and esophageal cancer treatment. Given the morbidity and mortality associated with the various treatments for esophageal cancer, it could be argued that HRQOL is as important an endpoint as survival, if not more so. Patient-reported pre-treatment HRQOL assessment appears to predict survival better than clinician-derived performance status assessment period. HRQOL assessment also appears to be responsive to surgical and non-surgical therapy and thus could potentially be used in trials and in practice to serve that function. Thus, HRQOL assessment could be a potentially important adjunct in shared decision-making and guiding treatment planning as well as monitoring the progress of treatment.
\end{abstract}

Keywords: Esophageal cancer; esophagectomy; health-related quality of life (HRQOL); patient-reported outcome

Submitted Aug 08, 2017. Accepted for publication Oct 27, 2017.

doi: $10.21037 /$ atm.2017.11.38

View this article at: http://dx.doi.org/10.21037/atm.2017.11.38

\section{Introduction}

The incidence of esophageal cancer has been increasing steadily in the past few decades; along with thyroid cancer, it has become the fastest rising solid tumor in the Western hemisphere $(1,2)$.

Esophagectomy is the mainstay of treatment for curative intent of local and locally-advanced esophageal cancer (3-5). Multimodality therapy including esophagectomy is the standard of care in locally-advanced cancer (6). Five-year overall survival is reported to be in the range of $20-40 \%(7-10)$. However, the procedure itself is complex and morbid with up to $10 \%$ postoperative mortality (6). Furthermore, the evidence suggests that up to $50 \%$ of patients will experience postoperative complications within the first month of

(c) Annals of Translational Medicine. All rights reserved. surgery (3-5). Recurrences can occur within the first year following surgery. These early recurrences are especially challenging and a source of distress for both patients and clinicians $(11,12)$. The select subgroup of patients who survive 5 years with no evidence of recurrence at that point have been reported to have life expectancies comparable to the age-matched general population $(5,13)$.

The difficulties associated with esophagectomy are mostly gastrointestinal in nature. Patients have problems with appetite loss, dysphagia, regurgitation, early satiety and consequently, weight loss along with other issues like diarrhea and fatigue $(2,14,15)$. Thus, the assessment of the effect of treatment on health-related quality of life (HRQOL) before, during and after treatment time is important for patients, caregivers and clinicians (15). 
Esophageal cancer itself has also been associated with poor HRQOL on initial presentation $(15,16)$. There is an association between pretreatment HRQOL and prognosis $(15,16)$. Previous studies show that higher HRQOL before treatment is associated with improved overall survival $(16,17)$. Thus, HRQOL is potentially helpful in guiding shared decision-making by physicians and patients.

This paper will review the evaluation of HRQOL in esophageal cancer patients undergoing curative intent therapy, the relationship between postoperative HRQOL and survival as well the potential utility of pre-treatment HRQOL as a prognostic tool.

\section{HRQOL surveys/instruments}

HRQOL instruments are classified into generic, general cancer and cancer-specific instruments $(18,19)$. These are typically referred to as patient-reported outcomes (PROs) or patient-reported outcome measures (PROMs). Different HRQOL instruments can be used as endpoints in research and each carry various advantages and disadvantages. One example is the Medical Outcomes Study Short Form (SF)-36 survey which is a PRO with 36 items that has been used to assess HRQOL in different diseases (19-23). It is useful when comparing HRQOL in a population with a known disease to the general population and in patients with different health conditions (20). The MOS SF-36 examines eight health domains: physical function, role physical, pain, general health perception, energy/vitality, social functioning, role emotional, and mental health $(20,21)$. It then combines those scores to result in two summary measures of physical and mental well-being $(20,21)$. Cancer-specific HRQOL instruments can be supplemented by other modules to assess aspects of the QOL that are commonly affected by the specific disease and associated treatment(s). The most commonly used tools for cancer are the European Organization for Research and Treatment of Cancer (EORTC) and the Functional Assessment of Cancer Therapy (FACT) (21). Those modules have been updated over the years and validated for the evaluation of patients with specific cancers, including esophageal cancer.

\section{Validated HRQOL tools for esophageal cancer}

The current version of the EORTC esophageal module was updated from the EORTC QLQ-OES24 developed in 1996. The 1996 EORTC esophageal module was developed after reviewing the literature followed by interviews conducted with patients and healthcare providers $(24,25)$. An initial 75 HRQOL issues were later refined to a list of 24 questions through a process of item reduction $(24,26)$. By 2003, validation studies conducted in 11 countries $(n=491)$ further reduced the list to 18 questions. The end result was the EORTC QOLQ-OES18 $(24,26)$. This esophageal module includes 4 domains: "dysphagia, eating, reflux and pain, and six single items: swallowing saliva, choking when swallowing, dry mouth, taste problems, coughing and speech problems" (24).

The Functional Assessment of Cancer Therapy-General (FACT-G) is used to evaluate the HRQOL in different cancers. It consists of 28 questions that cover the domains of "physical well-being, functional well-being, social wellbeing, and emotional well-being using a 5-point Likert scale" $(21,27)$. The FACT esophageal module validation process for esophageal cancer involved fewer patients when compared to the EORTC $(20,24)$. The esophageal cancer subscale (ECS) has 17 items that mainly explore two domains: swallowing and eating. It also included questions regarding the following: issues with breathing, chest pain on swallowing, mouth dryness, trouble swallowing, night cough, voice strength, communication with others, abdominal pain, good appetite, and weight loss $(20,24)$. The ECS was added to the FACT-G to create the FACT-E (e.g., FACT-G plus ECS = FACT-E) (20).

The EORTC tool covers two symptoms which the FACT-E does not: taste and reflux. On the other hand, the FACT system assesses weight loss while EORTC does not. Even when the domains cover the same general theme like eating, there are differences between both HRQOL tools. The QoLQ-OES18 contains 5 items in the eating domain (issues enjoying meals, early satiety, issues with eating in public, problems with taste) whereas the eating domain in FACT-E includes 3 items (eating liked food, enjoying meal with family and friends, eating food in the desired quantities). Thus, although it appears that both HRQOL tools are exploring similar domains, the argument could potentially be made that the $2 \mathrm{HRQOL}$ surveys may not be measuring exactly the same concepts or themes in the same way (24). The major difference between these 2 HRQOL surveys may be that whereas the EORTC survey reflects mainly physical symptoms and function, the FACT-E also reflects a psychosocial component. FACT-E was shown to be responsive to changes in esophageal cancer patient status over the course of treatment which makes it an excellent candidate to assess response to therapy in the context of trials $(20,24)$. 


\section{Postoperative HRQOL}

Systematic reviews have explored the utility of HRQOL tools in clinical decision-making for esophageal cancer patients $(28,29)$. The most recent review by Jacobs et al. included 62 articles, of which there were 6 RCTs, 12 cohort studies, 13 case series, one case control study and 30 cross-sectional studies. Sixty-five percent of those studies studied HRQOL as a primary outcome whereas thirty-four percent studied it as a secondary outcome (28). The EORTC QLQ-C30 was the most frequently used HRQOL questionnaire (28). The quality of the majority of those studies was poor. Only 4 studies had a robust methodology and only 26 studies met the criteria for outcome reporting. Two RCTs and one case series could be described as high quality studies. Adding to that, there is limited data about the effect of chemoradiotherapy or combined therapy and comparing open to minimally invasive surgery. Those deficiencies show the need for further studies in those areas. Despite the limitations of their review, Jacobs et al. recommended the use of HRQOL information from welldesigned studies in shared decision-making (28).

Derogar and Lagergren, in their 2012 prospective population-based cohort study, examined HRQOL in patients at 6 months, 3 and 5 years post esophagectomy using self-administered EORTC QLQ-C30 version 3.0 and EORTC QLQ-OES18 module (30). They compared HRQOL in esophageal cancer patients to age- and sex-matched general population (30). They reported that the majority of the patients who survived esophageal surgery to the 5 -year mark recovered to HRQOL levels comparable to the background, general population (30). Physical function was either stable or improved in $86 \%$ of patients, to a level which was similar to the general population (30). However, there was a subgroup of patients $(14 \%)$ that deteriorated substantially globally (30). This study had many strengths including a robust sample size, design, and the high survey response rate of $76 \%$ (30). However, it should be noted that the matching was done on a frequency basis rather than on an individual patient basis. An additional limitation is the possibility of a response shift causing an inflated level of HRQOL in esophageal cancer patients with 5-year survival as compared to the general population. In other words, there may be an adaptation mechanism of the perception of HRQOL as a result of a change in internal standards and values. This may lead to a perception of a new normal which might explain the improvement in the HRQOL scores (30). Although response shift can occur in the other direction as well (i.e., causing an apparent reduction in HRQOL), it is important to understand that this is a potential phenomenon when interpreting the results of HRQOL assessment at delayed time points. The authors reported that a further potential weakness in their study was the lack of baseline (preoperative) HRQOL scores (30). Courrech Staal et al. reported findings which appear to contradict those reported by Derogar and Lagergren (30). In their cross sectional study on HRQOL in long-term survivors, they included 163 consecutive patients and followed them up for a minimum of one year without recurrence (31). However, only 37 patients were included and 36 completed the QLQ-C30 and the QLQ-OES18 questionnaires. They were both compared to scores from a reference group of patients with esophageal cancer of all stages, including patients with loco-regional and distant recurrence. HRQOL scores were also compared with the scores of the general population without any adjustment according to demographics. The majority of the 36 patients received neoadjuvant therapy (mostly chemoradiotherapy) followed by surgery. The patients' scores showed improvements when compared to the reference group (31). However, when compared to the general population, the HRQOL scores were significantly worse than the scores of the general population (31). The study is limited by its cross sectional design, small sample size, high level of selection bias and lack of matching when comparing to the general population (31).

Djärv et al. used data derived from the previously discussed study (30) to study the relationship between baseline comorbidities of esophageal cancer patients and postoperative HRQOL (32). Several comorbidities were examined: cardiovascular [congestive heart failure (CHF) and angina], pulmonary conditions [asthma and chronic obstructive pulmonary disease (COPD)], diabetes along with liver disease, kidney disease, and elevated hypertension). Out of 153 patients who were alive at the 5 -year mark postoperatively, 141 patients completed the QLQ-C30 and the QLQ-OES18 questionnaires. Cardiovascular comorbidities were the most common comorbidities, followed by diabetes and pulmonary comorbidities. After esophagectomy, those with comorbidities had a statisticallyand clinically-significant worse global HRQOL at 6 months, 3 and 5 years as well as reports of significantly worse dyspnea and fatigue when compared to those without comorbidities (32). It was unclear whether there was a dose-response relationship such that increasing number of comorbidities was associated with decreasing HRQOL. 
The same authors also explored the effect of postoperative complications on postoperative HRQOL in another publication (5). The postoperative complications that were included in their analysis were complications that occurred during the first 30 days postoperatively; anastomotic leak, bleeding of 2 or more liters that required a return to the operating room, symptomatic or radiolograpically evident intraabdominal or intrathoracic abscess, pneumonia, sepsis, renal failure requiring dialysis, pulmonary embolism, myocardial infraction, stroke, and respiratory failure requiring intubation and ventilation. They used QLQ-C30 and the QLQ-OES18 measured at 3 months, 3 and 5 years after esophagectomy. Forty-six patients $(33 \%)$ had at least one complication postoperatively with respiratory complications (respiratory failure and pneumonia), leak and sepsis being the most common (5). Surprisingly, patients with at least one complication did not have significant differences in global HRQOL as compared to those patients without complications at all time-points (5). That being said, the dyspnea and fatigue domains were significantly worse at all 3 time-points in patients with complications. Furthermore, patients with complications reported significantly more eating restrictions than did patients without major postoperative complications but it was unclear whether this applied to all 3 time-points (5). Although not discussed in the report of that study, it is conceivable that anastomotic complications could result in both early and late (i.e., 6 months to 5 years) eating difficulties as anastomotic leaks can lead to anastomotic strictures. That study did not appear to specifically investigate the anastomotic complication subgroup. Patients with complications had worse progressive reflux at the 5 -year mark (5). The sensation of choking appeared to be transiently different between groups (5). The choking domain was significantly worse at the 6 -month mark for patients with complications but was not significantly different at 3 and 5 years after esophagectomy (5).

\section{HRQOL changes throughout therapy}

The trial outcome index (TOI) is calculated by summing three subscales from FACT-E: physical well-being (PWB), functional well-being and the ECS (15). The TOI score was used to evaluate HRQOL in esophageal cancer patients undergoing different treatment modalities in a longitudinal study by Trudel and colleagues (15). They included 84 patients who completed FACT-E questionnaires at baseline/pre-treatment and then at 1, 3, 6, 9, 12, 18, 24, and
36 months post-treatment. Forty-four patients underwent chemoradiation followed by surgery, 5 underwent chemotherapy followed by surgery, 20 were treated with surgery alone, 14 had definitive chemoradiation, and 1 patient received radiation therapy alone. Those who underwent preoperative chemotherapy or chemoradiation were further assessed at 6-8 weeks after initiating neoadjuvant therapy as well as after neoadjuvant therapy at about 12-14 weeks before surgery (15). HRQOL changed over the course of treatment. At the one-month mark, the HRQOL was worse across all the groups when compared to baseline $(\mathrm{P}<0.0001)$. Beyond the 3 -month mark, the HRQOL scores improved or stabilized. Comparing the treatment groups to surgery alone, it was noted that all combination treatments had an adverse effect on HRQOL scores. Thus, TOI is a measure of HRQOL that appears to be sensitive to changes throughout the course of treatment and may add important information to clinical decisionmaking, especially in patients undergoing multi-modality therapy (15).

A longitudinal prospective study by Parameswaran and colleagues evaluated the HRQOL in patients undergoing minimally invasive esophagectomy. HRQOL was assessed using the EORTC core questionnaire, EORTC QLQ-C30 (version 3.0), and with the esophageal site-specific module, EORTC QLQOES 18 at baseline within 3 weeks prior to surgery and at 6 weeks, 3, 6, and 12 months postoperatively (33). HRQOL was also assessed 2 weeks post-chemotherapy for those patients that received chemotherapy (33). Sixty-two patients underwent curative intent minimally invasive surgery with 48 patients undergoing chemotherapy (33). There were 2 conversions to open surgery (33). At the 6-week mark following surgery, there was a global deterioration of HRQOL which began to recover within the first three months reaching pre-treatment levels at 6 months which was maintained for a year postoperatively (33). Recovery was noted in all domains of HRQOL except for the reflux and diarrhea domains (33).

\section{Pre-treatment HRQOL as a prognostic tool}

Many studies have evaluated the post treatment HRQOL and its association with survival. Few studies looked into pre-treatment HRQOL and its use as a prognostic tool. Kidane et al. investigated this by pooling data from 4 prospective nonrandomized Canadian studies that included locally-advanced esophageal cancer who received neoadjuvant chemoradiotherapy or bimodality therapy 

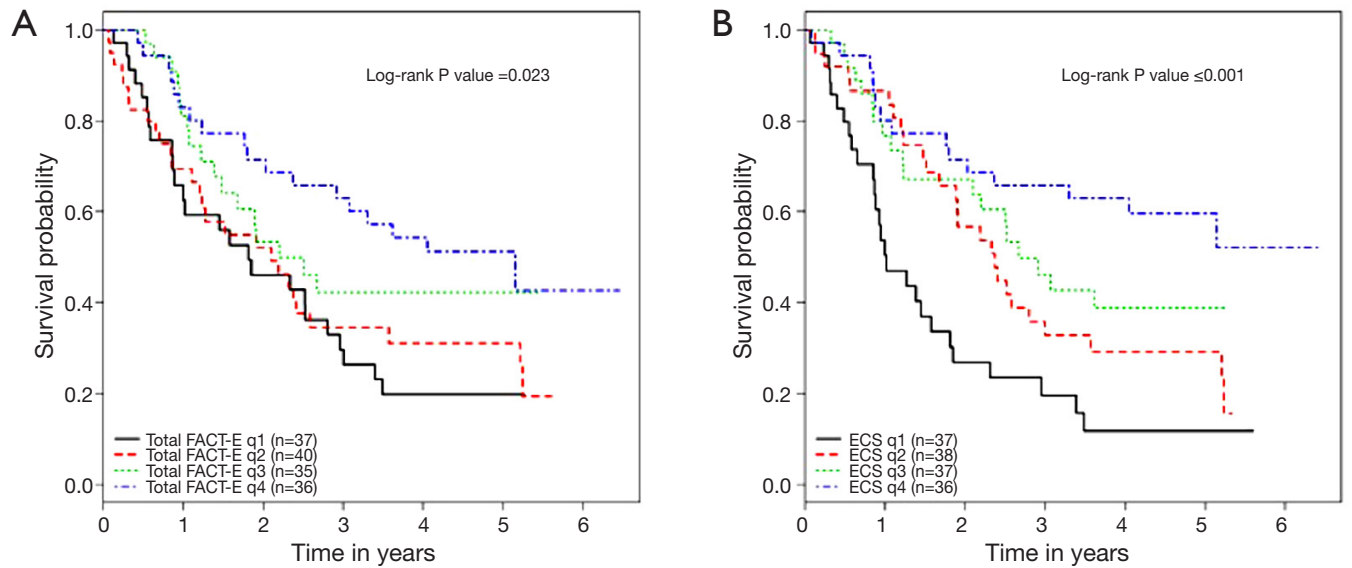

Figure 1 Overall survival increases as FACT-E \& ECS quartiles increase in patients with stage 2 and 3 esophageal cancer. (A) shows that FACT-E is significantly associated with overall survival while (B) describes a similar ECS association with overall survival (16). FACT-E, Functional Assessment of Cancer Therapy- Esophagus; ECS, esophageal cancer subscale.

without surgery (16). All patients completed pre-treatment FACT-E at the time of consultation and they were followed up after treatment was started every three months for the first two years then every 6 months until 5 years. All patients completed baseline FACT-E survey with $100 \%$ completion rate. Of 172 patients stage 2 and 3 patients treated with a curative intent, $62.3 \%$ of patients received pre/postoperative chemoradiotherapy (16). In their analyses, the authors controlled for possible confounders like age, histology, stage, surgery $v s$. no surgery, chemotherapy, radiation or the use of other adjuvant therapies, and the year of treatment (16). It was found that higher baseline FACT-E or ECS were both independently associated with better survival. Thus, pre-treatment HRQOL measures appeared to provide prognostication even when one controls for stage and treatments given $(9,16,34)$. ECS, with its focused and shorter number of questions, consistently showed a larger effect size and tighter confidence intervals than FACT-E. Thus, ECS may be a stronger predictor of overall survival when compared to FACT-E (16) (Figure 1). This study is limited by its small sample size and possible bias related to the use of clinical rather than pathological staging in patients who underwent definitive chemoradiation therapy (and thus did not have a final pathologic stage to report).

Kidane et al. also compared pretreatment FACT-E and ECS to clinician-assigned performance status (ECOG) in predicting survival in the same group of esophageal cancer patients (17). In this group of patients, 128 patients had available FACT-E and ECOG scores. On multivariable analysis where they controlled for the possible confounding effects of age, stage, histopathology, treatment year and types of treatment, it was found that pre-treatment FACT-E \& ECS were independently associated with overall survival whereas ECOG was not. Both FACT-E and ECS were able to discriminate between those who survived at 2 years and those who did not whereas ECOG could not adequately discriminate between survivors and non-survivors (17). The main message of this study was that patient-reported HRQOL was a better discriminator of overall survival than a score assigned by clinicians.

Blazeby and colleagues conducted one of the early studies that looked at pre-treatment HRQOL QOL (EORTC QLQ-C30 and EORTC QLQ-OES24) in a prospective study conducted from 1993-1995. They included 89 patients who were either treated surgically (before the neoadjuvant therapy era) or with palliative chemoradiotherapy. Those patients were followed until death occurred or until the end of the study. Only 38 patients were alive 6 months after treatment. They reported that higher physical function was associated with survival. This study is limited by its small sample size, the lack of control for important confounders such as the type of treatment received (35). Furthermore, this study was conducted in an era when neoadjuvant therapy was not commonly used, thus limiting its applicability to the modern era (35).

A randomized controlled trial by van Heijel et al. from 1994-2000 also explored pre-treatment HRQOL and its relationship to survival. They included 199 patients with adenocarcinoma who underwent surgery only without chemoradiotherapy. Patients completed pre-treatment 
Page 6 of 9

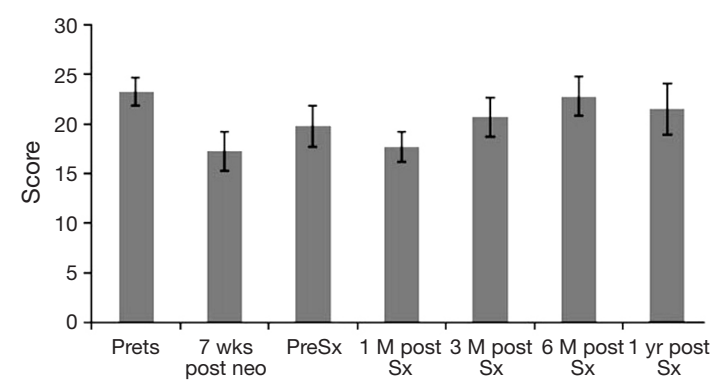

Figure 2 Physical well-being scores: pretreatment, 7 weeks post neoadjuvant therapy, pre-surgery, 1, 3, 6 months after surgery, and 1 year after surgery (9). wks, weeks; M, month; yr, year; Sx, surgery.

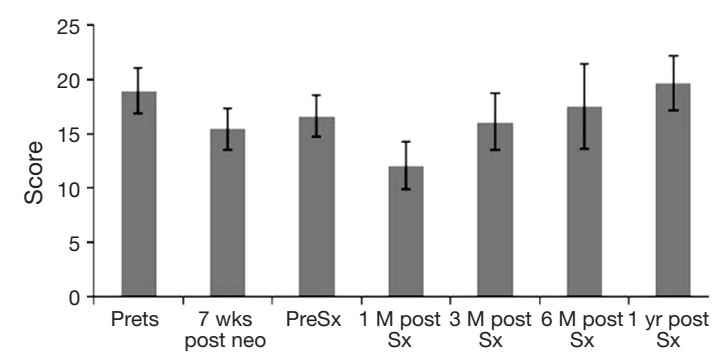

Figure 3 Functional well-being scores: pretreatment, 7 weeks after neoadjuvant therapy, pre-surgery, 1, 3, and 6 months after surgery, and 1 year after surgery (9). wks, weeks; M, month; yr, year; Sx, surgery.

HRQOL questionnaires 3 months preoperatively and 3 months postoperatively (Medical Outcome Study Short Form-20 and a modified but unvalidated Rotterdam Symptom Checklist). They reported that both the preoperative symptoms and the postoperative QOL subscales are predictors of survival in curable patients with adenocarcinoma. However, those results are not easily generalizable to esophageal cancer patients undergoing other modalities of treatment (36).

McKernan and colleagues also examined the relationship between quality of life (EORTC QLQ-C30) and survival in patients with gastroesophageal cancer (stages 1-3). In a study conducted between 1997 and 2002, 152 patients completed EORTC QLQ-C30 core questionnaires pretreatment; of these, 69 patients underwent a surgical intervention without neoadjuvant therapy whereas the rest underwent palliative or supportive care. Multivariable survival analysis showed that tumor stage, treatment, and the appetite loss component of the EROTC QLQ-C30 but not the ECOG were significantly associated with survival in gastroesophageal cancer patients (37)

Quinten and colleagues conducted an extensive analysis on this topic using data from 30 RCTs from 1986-2004 that studied different cancer sites, including esophageal cancer. EORTC QLQ-C30 questionnaires were completed at baseline with low compliance (65\%). Quinten et al. reported that for each of the 11 studied cancer sites, at least 1 HRQOL domain had prognostic value globally. However, esophageal cancer was one of the few cancers in which Quinten $e t a l$. were able to demonstrate that pre-treatment HRQOL was associated with survival (38).

\section{Return to baseline HRQOL after therapy}

It is helpful for patients and clinicians to understand the impact of therapy on patients' HRQOL. It is also helpful to understand the expected trajectory of HRQOL as treatment progresses so that clinicians can more effectively inform patients about the estimated time it may take to return to their baseline level of HRQOL. Chemoradiation therapy appears to have a transient yet significant effect on the HRQOL in esophageal cancer patients; however, HRQOL then returns to baseline/pre-treatment levels 5-7 weeks post-treatment (9) (Figures 2,3). In an RCT that compared definitive chemoradiation to chemoradiation followed by surgery, HRQOL was significantly worse in the first 3 months in patients who underwent surgery. This changed at the 6 months mark as HRQOL scores became comparable to baseline/pre-treatment levels. HRQOL continued to improve in surgical patients with time $(9,39)$.

Surgery is known to have an immediate effect on HRQOL. However, it returns back to baseline within 3 months. At one year, HRQOL continues to improve when compared to preoperative baseline (9). It is important to note that although global/overall HRQOL appears to return to baseline/pre-treatment levels, the physical functioning component of HRQOL still remains poorer or worse than baseline/pre-treatment levels (9). This serves to enhance the face validity to these results. Parameswaran and colleagues reported similar finding of transient deterioration of the HRQOL 6 weeks after surgery that starts to improve within 3 months and returns to baseline at 6 months (33).

\section{Open vs. minimally invasive esophagectomy (MIE)}

One RCT examined the short-term effects of MIE (40). They reported less pain, blood loss, and pulmonary infections postoperatively (40). They also reported a better HRQOL at 
Table 1 Comparison of HRQOL measures between open and minimally-invasive (41)

\begin{tabular}{|c|c|c|c|}
\hline HRQOL measure & OE & MIE & $P$ value \\
\hline \multicolumn{4}{|l|}{ SF $36^{\dagger}$} \\
\hline \multicolumn{4}{|c|}{ Mental component summary } \\
\hline Preoperatively & $45[9 ; 43-48]$ & $46[12 ; 43-49]$ & 0.955 \\
\hline 6 weeks & $45[11 ; 40-50]$ & $46[10 ; 41-50]$ & 0.806 \\
\hline 1 year & $50[10 ; 47-53]$ & $53[10 ; 49-56]$ & 0.317 \\
\hline \multicolumn{4}{|c|}{ Physical component summary } \\
\hline Preoperatively & $43[9 ; 40-46]$ & $46[8 ; 44-48]$ & 0.072 \\
\hline 6 weeks & 36 [6; 34-39] & $42[8 ; 39-46]$ & 0.007 \\
\hline 1 year & $45[9 ; 42-48]$ & $50[6 ; 48-53]$ & 0.003 \\
\hline \multicolumn{4}{|l|}{ EORTC $\mathrm{C} 30^{\dagger}$} \\
\hline \multicolumn{4}{|l|}{ Global health } \\
\hline Preoperatively & $63[23 ; 56-70]$ & $66[22 ; 60-72]$ & 0.631 \\
\hline 6 weeks & $51[21 ; 44-58]$ & $61[18 ; 56-67]$ & 0.02 \\
\hline 1 year & $67[21 ; 60-75]$ & 79 [10; 76-83] & 0.042 \\
\hline \multicolumn{4}{|l|}{ EORTC OES $18^{\ddagger}$} \\
\hline \multicolumn{4}{|l|}{ Pain } \\
\hline Preoperatively & $23[17-22,22-30]$ & $17[24 ; 11-24]$ & 0.187 \\
\hline 6 weeks & $19[13-21,21-26]$ & $8[11 ; 5-11]$ & 0.002 \\
\hline 1 year & $16[16 ; 10-22]$ & $6[9 ; 3-10]$ & 0.003 \\
\hline \multicolumn{4}{|l|}{ Talking } \\
\hline Preoperatively & $12[25 ; 4-19]$ & $10[23 ; 4-17]$ & 0.745 \\
\hline 6 weeks & 37 [39; 25-49] & $18[26 ; 10-26]$ & 0.008 \\
\hline 1 year & 10 [21; 3-18] & $5[14 ; 0-11]$ & 0.288 \\
\hline
\end{tabular}

Data are presented as mean $(\mathrm{SD}, 95 \% \mathrm{Cl}) .{ }^{\dagger}$, measure general health aspects: better well-being is associated with higher scores; ${ }^{\ddagger}$, measures esophageal functions: lower scores are associated with better function. OE, open esophagectomy; MIE, minimally invasive esophagectomy; EORTC, European Organization for Research and Treatment of Cancer; HRQOL, health-related quality of life; SF 36, short form 36 health survey.

6 weeks postoperatively compared to open esophagectomy (40). HRQOL in open vs. MIE was also examined in an international multicenter RCT from 2009-2011 with a one year follow up. QOL was assessed by SF 36 and EORTC C30 and OES18 tools at baseline, 6 weeks, and at 1 year postoperatively. At one year, the QOL was better in both groups when compared at six weeks and baseline. Moreover, the MIE group had better scores at one year when compared to the open esophagectomy group in three domains: global health, pain, and physical activity (41) (Table 1).

Kauppila and colleagues conducted the most recent systemic review that included 9 studies comparing open surgery to minimally invasive surgery. Of 2,064 patients, $1,157(56.1 \%)$ underwent minimally invasive surgery and 907 (43.9\%) underwent open surgery. The HRQOL outcomes were assessed using the EORTC QLQ-C30 and the QLQ-OES18 questionnaires in eight studies. Their meta-analysis of these outcomes showed that patients who underwent minimally invasive surgery had better outcomes at 4-6 weeks. At three months, patients had better global QOL, physical function, less fatigue, and better pain control. However, no clinically significant difference noted after 6 months except for better physical function in patients who underwent minimally invasive surgery (42).

\section{Conclusions}

While esophagectomy is the mainstay of curative therapy for esophageal cancer, it is associated with significant morbidity and mortality, with subsequent major impact on quality of life. HRQOL assessment is valuable in helping clinicians understand the impact on patients of esophageal cancer and the various treatments thereof. HRQOL is also valuable as an end-point in studies of esophageal cancer and esophageal cancer treatment. Given the morbidity and mortality associated with the various treatments for esophageal cancer, it could be argued that HRQOL is as important an endpoint as survival, if not more so. Patientreported pre-treatment HRQOL assessment appears to predict survival better than clinician-derived performance status assessment HRQOL assessment also appears to be responsive to surgical and non-surgical therapy and thus could potentially be used to in trials and in practice to serve that function. Thus, HRQOL assessment could be a potentially important adjunct in shared decision-making and guiding the treatment planning as well as monitoring the progress of treatment.

\section{Acknowledgements}

None.

\section{Footnote}

Conflicts of Interest: The authors have no conflicts of interest 
to declare.

\section{References}

1. Jemal A, Murray T, Samuels A, et al. Cancer statistics, 2003. CA Cancer J Clin 2003;53:5-26.

2. Scarpa $M$, Valente $S$, Alfieri R, et al. Systematic review of health-related quality of life after esophagectomy for esophageal cancer. World J Gastroenterol 2011;17:4660-74.

3. Viklund P, Lindblad M, Lu M, et al. Risk factors for complications after esophageal cancer resection: A prospective population-based study in Sweden. Ann Surg 2006;243:204-11.

4. Daly JM, Fry WA, Little AG, et al. Esophageal cancer: results of an American College of Surgeons Patient Care Evaluation Study. J Am Coll Surg 2000;190:562-72.

5. Derogar M, Orsini N, Sadr-Azodi O, et al. Influence of major postoperative complications on health-related quality of life among long-term survivors of esophageal cancer surgery. J Clin Oncol 2012;30:1615-9.

6. Kidane B, Coughlin S, Vogt K, et al. Preoperative chemotherapy for resectable thoracic esophageal cancer. Cochrane Database Syst Rev 2015;(5):CD001556.

7. Orringer MB, Marshall B, Chang AC, et al. Two thousand transhiatal esophagectomies. Ann Surg 2007;246:363-72; discussion 372-4.

8. Reynolds JV, McLaughlin R, Moore J, et al. Prospective evaluation of quality of life in patients with localized oesophageal cancer treated by multimodality therapy or surgery alone. Br J Surg 2006;93:1084-90.

9. Safieddine N, Xu W, Quadri SM, et al. Health-related quality of life in esophageal cancer: effect of neoadjuvant chemoradiotherapy followed by surgical intervention. J Thorac Cardiovasc Surg 2009;137:36-42.

10. Chang YL, Tsai YF, Chao YK, et al. Quality-of-life measures as predictors of post-esophagectomy survival of patients with esophageal cancer. Qual Life Res 2016;25:465-75.

11. Stiles BM, Salzler GG, Nasar A, et al. Clinical predictors of early cancer-related mortality following neoadjuvant therapy and oesophagectomy. Eur J Cardiothorac Surg 2015;48:455-60.

12. Kidane B, Darling GE. Improving decision-making regarding oesophagectomy after preoperative therapy. Eur J Cardiothorac Surg 2015;48:461-2.

13. Rouvelas I, Zeng W, Lindblad M, et al. Survival after surgery for oesophageal cancer: A populationbased study. Lancet Oncol 2005;6:864-70.
14. Viklund P, Wengström Y, Rouvelas I, et al. Quality of life and persisting symptoms after oesophageal cancer surgery. Eur J Cancer 2006;42:1407-14.

15. Trudel JG, Sulman J, Atenafu EG, et al. Longitudinal evaluation of trial outcome index scores in patients with esophageal cancer. Ann Thorac Surg 2016;102:269-75.

16. Kidane B, Sulman J, Xu W, et al. Baseline measure of health-related quality of life (Functional Assessment of Cancer Therapy-Esophagus) is associated with overall survival in patients with esophageal cancer. J Thorac Cardiovasc Surg 2016;151:1571-80.

17. Kidane B, Sulman J, Xu W, et al. Pretreatment quality-oflife score is a better discriminator of oesophageal cancer survival than performance status. Eur J Cardiothorac Surg 2017;51:148-54.

18. Lipscomb J, Gotay CC, Snyder CF. Patient-reported outcomes in cancer: a review of recent research and policy initiatives. CA Cancer J Clin 2007;57:278-300.

19. Ware JE Jr, Sherbourne CD. The MOS 36-item shortform health survey (SF-36). I. Conceptual framework and item selection. Med Care 1992;30:473-83.

20. Darling G, Eton DT, Sulman J, et al. Validation of the functional assessment of cancer therapy esophageal cancer subscale. Cancer 2006;107:854-63.

21. Darling GE. Quality of life in patients with esophageal cancer. Thorac Surg Clin 2013;569-75

22. McHorney CA, Ware JE Jr, Raczek AE. The MOS 36item Short-Form Health Survey (SF-36): II. Psychometric and clinical tests of validity in measuring physical and mental health constructs. Med Care 1993;31:247-63.

23. McHorney CA, Ware JE Jr, Lu JF, et al. The MOS 36 item Short-Form Health Survey (SF-36): III. Tests of data quality, scaling assumptions, and reliability across diverse patient groups. Med Care 1994;32:40-66.

24. McNair AG, Blazeby JM. Health-related quality-of-life assessment in GI cancer randomized trials: improving the impact on clinical practice. Expert Rev Pharmacoecon Outcomes Res 2009;9:559-67.

25. Blazeby JM, Alderson D, Winstone K, et al. Development of a EORTC questionnaire module to be used in quality of life assessment for patients with oesophageal cancer. Eur J Cancer 1996;32:1912-7.

26. Blazeby JM, Conroy T. Hammerlid E, et al. Clinical and psychometric validation of an EORTC questionnaire module, the EORTC QLQ-OES18, to assess quality of life in patients with oesophageal cancer. Eur J Cancer 2003;39:1384-94.

27. Cella DF, Tulsky DS, Gray G, et al. The functional 
assessment of cancer therapy scale: development and validation of the general measure. J Clin Oncol 1993;11:570-9.

28. Jacobs M, Macefield RC, Blazeby JM, et al. Systematic review reveals limitations of studies evaluating healthrelated quality of life after potentially curative treatment for esophageal cancer. Qual Life Res 2013;22:1787-803.

29. Parameswaran R, McNair A, Avery KN, et al. The role of health related quality of life outcomes in clinical decision making in surgery for esophageal cancer: a systematic review. Ann Surg Oncol 2008;15:2372-9.

30. Derogar M, Lagergren P. Health-related quality of life among 5-year survivors of esophageal cancer surgery: a prospective population-based study. J Clin Oncol 2012;30:413-8.

31. Courrech Staal EF, van Sandick JW, van Tinteren H, et al. Health-related quality of life in long-term esophageal cancer survivors after potentially curative treatment. J Thorac Cardiovasc Surg 2010;140:777-83.

32. Djärv T, Derogar M, Lagergren P. Influence of co-morbidity on long-term quality of life after oesophagectomy for cancer. Br J Surg 2014;101:495-501.

33. Parameswaran R, Blazeby JM, Hugues R, et al. Health related quality of life after minimally invasive oesophagectomy. Br J Surg 2010;97:525-31

34. Djärv T, Lagergren P. Six-month postoperative quality of life predicts long-term survival after oesophageal cancer surgery. Eur J Cancer 2011;47:530-5.

35. Blazeby JM, Brookes ST, Alderson D. The prognostic value of quality of life scores during treatment for oesophageal cancer. Gut 2001;49:227-30.

Cite this article as: Alghamedi A, Buduhan G, Tan L, Srinathan SK, Sulman J, Darling G, Kidane B. Quality of life assessment in esophagectomy patients. Ann Transl Med 2018;6(4):84. doi: 10.21037/atm.2017.11.38
36. van Heijl M, Sprangers MA, de Boer AG, et al.

Preoperative and early postoperative quality of life predict survival in potentially curable patients with esophageal cancer. Ann Surg Oncol 2010;17:23-30.

37. McKernan M, McMillan DC, Anderson JR, et al. The relationship between quality of life (EORTC QLQ-C30) and survival in patients with gastro-oesophageal cancer. $\mathrm{Br}$ J Cancer 2008;98:888-93.

38. Quinten C, Martinelli F, Coens C, et al. A global analysis of multitrial data investigating quality of life and symptoms as prognostic factors for survival in different tumor sites. Cancer 2014;120:302-11.

39. Bonnetain F, Bouché O, Michel P, et al. A comparative longitudinal quality of life study using the Spitzer quality of life index in a randomized multicenter phase III trial (FFCD9102): chemoradiation followed by surgery compared with chemoradiation alone in locally advanced squamous resectable thoracic esophageal cancer. Ann Oncol 2006;17:827-34.

40. Biere SS, van Berge Henegouwen MI, Maas KW, et al. Minimally invasive versus open oesophagectomy for patients with oesophageal cancer: a multicentre, open-label, randomised controlled trial. Lancet 2012;379:1887-92.

41. Maas KW, Cuesta MA, van Berge Henegouwen MI, et al. Quality of life and late complications after minimally invasive compared to open esophagectomy: results of a randomized trial. World J Surg. 2015;39:1986-93.

42. Kauppila JH, Xie S, Johar A, et al. Meta-analysis of healthrelated quality of life after minimally invasive versus open oesophagectomy for oesophageal cancer. Br J Surg 2017;104:1131-40. 